\title{
COEFFICIENTS OF FUNCTIONS WITH BOUNDED BOUNDARY ROTATION
}

\author{
JAMES W. NOONAN ${ }^{1}$
}

ABstract. For $k \geqq 2$ denote by $V_{k}$ the class of normalized functions analytic in the unit disc which have boundary rotation at most $k \pi$. For fixed $n \leqq(k+6) / 4$ we determine the maximum of the set of values of $\left|a_{n}\right|$, where $a_{n}$ is the $n$th Taylor coefficient of a function in $V_{k}$.

For a fixed $k \geqq 2$ let $V_{k}$ denote the class of functions

$$
f(z)=z+a_{2} z^{2}+a_{3} z^{3}+\cdots
$$

which are analytic in $U=\{z:|z|<1\}$ and have an integral representation of the form

$$
f^{\prime}(z)=\exp \left\{\frac{1}{\pi} \int_{0}^{2 \pi} \log \left(1-z e^{-i t}\right)^{-1} d \mu(t)\right\},
$$

where $\mu(t)$ is real-valued and of bounded variation on $[0,2 \pi]$ with

$$
\int_{0}^{2 \pi} d \mu(t)=2 \pi, \quad \int_{0}^{2 \pi}|d \mu(t)| \leqq k \pi .
$$

V. Paatero [7] showed that $f(z)$ given by (1) belongs to $V_{k}$ if and only if $f^{\prime}(z) \neq 0$ in $U$ and $f(z)$ maps $U$ onto a domain with boundary rotation at most $k \pi$. (See [4] for a definition of this concept.)

$V_{2}$ is precisely the class of normalized univalent functions that map $U$ onto a convex domain, and it is known [7] that for $2 \leqq k \leqq 4, V_{k}$ consists only of univalent functions. It is also known [1] that $f(z) \in V_{k}$ if and only if there exist $s_{1}(z)$ and $s_{2}(z) \in S^{*}$, the class of normalized starlike functions, such that

$$
f^{\prime}(z)=\frac{\left(s_{1}(z) / z\right)^{(k+2) / 4}}{\left(s_{2}(z) / z\right)^{(k-2) / 4}} .
$$

Presented to the Society, January 23, 1971; received by the editors November 2, 1970.

AMS 1970 subject classifications. Primary 30A34, 30A32; Secondary 30A40.

Key words and phrases. Coefficients, bounded boundary rotation, convex functions, starlike functions.

${ }^{1}$ The results in this paper are part of the author's Ph.D. thesis, written at the University of Maryland under the direction of Professor W. E. Kirwan. The author at present holds a National Research Council Postdoctoral Resident Research Associateship at the Naval Research Laboratory, Washington, D. C.

Copyright (c) 1971, American Mathematical Society 
This follows directly from (2).

In spite of considerable effort, the problem of determining $M(n, k)$ $=\max _{f \in V_{k}}\left|a_{n}\right|$ where $f(z)$ is given by (1) has been solved for all $n$ only when $k=2$ and $k=4$. K. Löwner [6] proved that $M(n, 2)=1$, and A. Rényi [8] proved that $M(n, 4)=n$. O. Lehto [4] proved that $M(2, k)=k / 2$ and $M(3, k)=\left(k^{2}+2\right) / 6$. It is also known [2] that $M(4, k)=\left(k^{3}+8 k\right) / 24$. The extremal function in all of these cases has been

$$
F_{k}(z)=\frac{1}{k}\left\{\left(\frac{1+z}{1-z}\right)^{k / 2}-1\right\} .
$$

It is also known $([3],[9])$ that $M(n, k) \leqq C(k) n^{k / 2-1}$ where $C(k)$ depends only on $k$, and [4] that

$$
M(n, k) \sim k^{n-1} / n ! \quad(k \rightarrow \infty) .
$$

Again $F_{k}(z)$ is extremal. These facts tend to support the conjecture that $F_{k}(z)$ is the solution of the coefficient problem $M(n, k)$ for all $n$ and for all $k$. The purpose of this paper is to show that if $n \leqq[(k+6) / 4]$, then $F_{k}(z)$ is the solution to the problem $M(n, k)$.

Before we can prove this, however, we need two lemmas. The proofs of these lemmas are modeled after that of Lemma 4 [5, p. 169], which in turn is due to Professor M.S. Robertson.

LEMmA 1. Let $s(z) \in S^{*}$ and let $\beta>0$. Let $g(z)=(s(z) / z)^{-\beta}=$ $\sum_{n=0}^{\infty} b_{n} z^{n}$. Then for $0 \leqq n \leqq \beta+1$ we have

$$
\left|b_{n}\right| \leqq \frac{\Gamma(2 \beta+1)}{\Gamma(n+1) \Gamma(2 \beta-n+1)},
$$

where $\Gamma$ represents the gamma function.

Proof. From the definition of $g(z)$ we have

$$
\log g(z)=-\beta \log \frac{s(z)}{z} .
$$

By differentiating both sides of this equation, we see that

$$
1-z g^{\prime}(z) / \beta g(z)=z s^{\prime}(z) / s(z) .
$$

Since $s(z) \in S^{*}$, we see that

$$
P(z)=1-z g^{\prime}(z) / \beta g(z)
$$

belongs to $P$, the class of normalized functions with positive real part. Therefore, $(P(z))^{-1}=1+\sum_{m=1}^{\infty} \mu_{m} z^{m} \in \mathcal{P}$, and $\left|\mu_{m}\right| \leqq 2$ for all $m$. 
Now $(P(z))^{-1}\left(\beta g(z)-z g^{\prime}(z)\right)=\beta g(z)$. Thus by equating coefficients of $z^{n}$ we see that

$$
n b_{n}=\sum_{m=0}^{n-1}(\beta-m) b_{m} \mu_{n-m} .
$$

A simple induction argument, in which we use the fact that $\left|\mu_{n-m}\right| \leqq 2$, now completes the proof. The requirement that $n \leqq \beta+1$ is used in the induction argument to insure that $\beta-(n-1) \geqq 0$.

Lemma 2. Let $s(z) \in S^{*}$ and let $\alpha>0$. Let $g(z)=(s(z) / z)^{\alpha}=$ $\sum_{n=0}^{\infty} b_{n} z^{n}$. Then

$$
\left|b_{n}\right| \leqq \frac{\Gamma(n+2 \alpha)}{\Gamma(n+1) \Gamma(2 \alpha)} .
$$

Proof. As in the proof of Lemma 1 we see that

$$
\log g(z)=\alpha \log \frac{s(z)}{z},
$$

which shows that

$$
P(z)=1+\frac{z g^{\prime}(z)}{\alpha g(z)}=1+\sum_{n=1}^{\infty} \delta_{n} z^{n}
$$

belongs to $\odot$. Thus $\left|\delta_{n}\right| \leqq 2$.

By expanding $g(z)$ and $g^{\prime}(z)$ as power series and then comparing coefficients of $z^{n}$, we see that

$$
n b_{n}=\alpha \sum_{m=0}^{n-1} b_{m} \delta_{n-m} .
$$

The proof is then completed by induction. Note that this lemma is true for all $n$.

We are now able to prove the following theorem on the coefficients of a $V_{k}$ function.

TheOREM. Let $f(z)=z+\sum_{n=2}^{\infty} a_{n} z^{n} \in V_{k}$, and let $F_{k}(z)=z+$ $\sum_{n=2}^{\infty} A_{n}(k) z^{n}$ be given by (4). Then for $n \leqq[(k+6) / 4]$ we have $\left|a_{n}\right|$ $\leqq\left|A_{n}(k)\right|$ with equality for any $n \leqq[(k+6) / 4]$ if and only if $f(z)$ $=e^{i \theta} F_{k}\left(e^{-i \theta} z\right)$ for some $\theta \in[0,2 \pi]$.

Proof. By (3) we have that

$$
f^{\prime}(z)=\frac{\left(s_{1}(z) / z\right)^{(k+2) / 4}}{\left(s_{2}(z) / z\right)^{(k-2) / 4}}
$$


where $s_{1}(z), s_{2}(z) \in S^{*}$. Let

$$
\left(s_{1}(z) / z\right)^{(k+2) / 4}=\sum_{j=0}^{\infty} c_{j} z^{j}
$$

and

$$
\left(s_{2}(z) / z\right)^{-(k-2) / 4}=\sum_{j=0}^{\infty} b_{j} z^{j}
$$

Then

$$
f^{\prime}(z)=\sum_{m=1}^{\infty} m a_{m} z^{m-1}=\left(\sum_{j=0}^{\infty} c_{j} z^{j}\right)\left(\sum_{h=0}^{\infty} b_{h} z^{h}\right) .
$$

By equating coefficients we see that

$$
(n+1) a_{n+1}=c_{0} b_{n}+c_{1} b_{n-1}+\cdots+c_{n-1} b_{1}+c_{n} b_{0} .
$$

From Lemma 2, with $\alpha=(k+2) / 4$, we have that

$$
\left|c_{j}\right| \leqq \frac{\Gamma(j+k / 2+1)}{\Gamma(j+1) \Gamma(k / 2+1)} \quad(j \geqq 0)
$$

and from Lemma 1 , with $\beta=(k-2) / 4$, we have that

$$
\left|b_{m}\right| \leqq \frac{\Gamma(k / 2)}{\Gamma(m+1) \Gamma(k / 2-m)} \quad(0 \leqq m \leqq(k+2) / 4) .
$$

Therefore, for $n \leqq[(k+2) / 4]$ we have that

$$
(n+1)\left|a_{n+1}\right| \leqq \sum_{j=0}^{n} \frac{\Gamma(j+k / 2+1)}{\Gamma(j+1) \Gamma(k / 2+1)}
$$

$$
\frac{\Gamma(k / 2)}{\Gamma(n-j+1) \Gamma(k / 2-(n-j))} \cdot
$$

Now let us examine

$$
F_{k}^{\prime}(z)=\frac{(1-z)^{-(k+2) / 2}}{(1+z)^{-(k-2) / 2}}
$$

Let $(1-z)^{-(k+2) / 2}=\sum_{n=0}^{\infty} C_{n} z^{n}$ and $(1+z)^{(k-2) / 2}=\sum_{m=0}^{\infty} B_{m} z^{m}$. Then

$$
C_{n}=\frac{\Gamma(n+k / 2+1)}{\Gamma(n+1) \Gamma(k / 2+1)} \quad(n \geqq 0)
$$

and 


$$
B_{n}=\frac{\Gamma(k / 2)}{\Gamma(n+1) \Gamma(k / 2-n)} \quad(0 \leqq n \leqq k / 2) .
$$

Then we see that

$$
(n+1) A_{n+1}(k)=C_{0} B_{n}+C_{1} B_{n-1}+\cdots+C_{n-1} B_{1}+C_{n} B_{0} .
$$

Since we require that $n \leqq[(k+2) / 4]$, we have also that $n \leqq[k / 2]$. Therefore

$$
\begin{aligned}
& (n+1) A_{n+1}(k) \\
& \quad=\sum_{j=0}^{n} \frac{\Gamma(j+k / 2+1)}{\Gamma(j+1) \Gamma(k / 2+1)} \\
& \quad \cdot \frac{\Gamma(k / 2)}{\Gamma(n-j+1) \Gamma(k / 2-(n-j))} \quad(n \leqq[(k+2) / 4]) .
\end{aligned}
$$

Comparing (6) with (7), we find that

$$
\left|a_{n+1}\right| \leqq\left|A_{n+1}(k)\right| \quad(n \leqq[(k+2) / 4]) .
$$

This is equivalent to the statement of the theorem.

If equality holds for any $n$, then an examination of the above proof shows that $\left|a_{2}\right|=A_{2}(k)=k / 2$. But it is well-known [4] that $\left|a_{2}\right|=k / 2$ if and only if $f(z)=e^{i \theta} F_{k}\left(e^{-i \theta} z\right)$ for some $\theta \in[0,2 \pi]$. This completes the proof of the theorem.

This theorem allows us to improve Lehto's result (5) in the following sense.

Corollary. Let $F_{k}(z)=z+\sum_{n=2}^{\infty} A_{n}(k) z^{n}$ be given by (4). Let $n$ be fixed but arbitrary, $n \geqq 2$. Then for $k \geqq 4 n-6$ we have $M(n, k)=A_{n}(k)$. In particular, for any fixed $n, M(n, k) \sim k^{n-1} / n !(k \rightarrow \infty)$.

Proof. Since $k \geqq 4 n-6$, we have $n \leqq[(k+6) / 4]$. Then, by the theorem, we see that $M(n, k)=A_{n}(k)$. Direct computation then shows that $M(n, k) \sim k^{n-1} / n !(k \rightarrow \infty)$.

\section{BIBLIOGRAPHY}

1. D. A. Brannan, On functions of bounded boundary rotation. I, Proc. Edinburgh Math. Soc. 16 (1968/69), 339-347.

2. - On functions of bounded boundary rotation. II, Bull. London Math. Soc. 1 (1969), 321-322.

3. W. E. Kirwan, On the coefficients of functions with bounded boundary rotation, Michigan Math. J. 15 (1968), 277-282. MR 38 \#1250.

4. O. Lehto, On the distortion of conformal mappings with bounded boundary rotation, Ann. Acad. Sci. Fenn. Ser. A I Math.-Phys. No. 124 (1952). MR 14, 743. 
5. A. E. Livingston, p-valent close-to-convex functions, Trans. Amer. Math. Soc. 115 (1965), 161-179. MR 33 \#7520.

6. K. Löwner, Untersuchungen über die Verzerrung bei konformen Abbildungen des Einheitskreises $|z|<1$, die durch Funktionen mit nicht verschwindender Ableitung geliefert werden, Leipzig Berichte 69 (1917), 89-106.

7. V. Paatero, Über die konforme Abbildung von Gebieten deren Ränder von beschränkter Drehung sind, Ann. Acad. Sci. Fenn. Ser. A No. 9 (1931).

8. A. Rényi, On the coefficients of schlicht functions, Publ. Math. Debrecen 1 (1949), 18-23. MR 11, 92.

9. M. S. Robertson, Coefficients of functions with bounded boundary rotation, Canad. J. Math. 21 (1969), 1477-1482. MR 41 \#458.

University of Maryland, College Park, Maryland 20742

E. O. Hulburt Center for Space Research, Naval Research Laboratory, WASHINGTON, D. C. 